\title{
The Method of Avoidance of Unmanned Vehicles in Dynamic Environment
}

\author{
Pingli Ren \\ Changzhou Vocational Institute of Mechatronic Technology, Changzhou, Jiangsu, 213164, China
}

Keywords: Dynamic Environment, Unmanned Fleet, Obstacle Avoidance Method, Policy Strategy

\begin{abstract}
With the rapid development of economy and technology in China, driverless cars have gradually entered the eyes of people and have been widely concerned by the society. driverless car is a kind of land-type intelligent robot. as the name implies, driverless car does not need manpower to operate during operation. it can pass through the computer inside the driverless car. the intelligent driver with computer system as the core is the core of the driverless car. The unmanned vehicle is equipped with the on-board sensing system, which can capture the links around the driverless vehicle in the first time, and analyze the information obtained to realize the automatic planning of the vehicle route.
\end{abstract}

\section{An Overview of Driverless Cars and Their Significance}

The introduction of driverless cars is an inevitable trend of social development in our country, which can solve the hidden safety problems in the course of operation efficiently, prevent the hidden trouble for automobile safety due to the discomfort of drivers, and bring some hidden dangers to the modern development of our country. The following will focus on approaches to obstacle avoidance for unmanned convoys in dynamic environments.

In recent years, according to the investigation and research, the number of people's vehicles in China is not less than 150 million, and there are more than one million vehicles caused by traffic accidents, the number of deaths is 2.22, the emergence of traffic accidents has brought a greater security threat to our people. And driverless cars are an important way to solve the traffic safety problems in China, which has an important impact on the development of our transportation system. Based on the tool point of view, driverless cars in the larger representative intelligent robot, driverless cars can reach their destination from a set starting point, and in the process of driving according to the actual situation of the path, to complete the realization of road conditions obstacle avoidance, through intelligent analysis can get the best driving path, to achieve efficient and safe driving. In fact, for a driverless car, its core equipment is the intelligent driver in the driverless car, the operation of the driverless car is controlled by the intelligent driver, by the driver-only driver to ensure the safety of the driverless car. The driving instrument of driverless car mainly takes the computer system as the core technology to realize the guarantee function for the driverless car. Not only that, if we want to realize the multiple functional approaches in driverless cars, it is far from enough to rely on intelligent driving instruments alone, but also need to cooperate with other technologies on driverless cars. Of course, it is not doubtful that for driverless cars, computer technology is the main core technology of driverless cars. In the process of realizing obstacle avoidance, driverless vehicles can be divided into the following three parts: first, the technology of driverless vehicles will take the lead in detecting the surrounding environment of driverless vehicles and analyzing the detected obstacles. Finally, the driverless car will avoid obstacles according to the content of analysis and prediction, this function mainly depends on the path decision system of the driverless car analysis. 


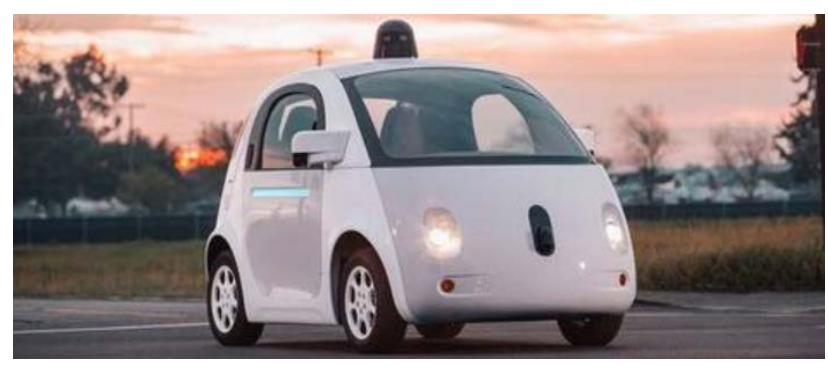

Figure 1 Driverless vehicles

\section{Circumvention Unmanned Convoys}

\subsection{Common Forms of Vehicle Avoidance}

In recent years, there have been more profound research on driverless cars at home and abroad, and a deeper understanding of the successful obstacle avoidance of driverless car fleets. In general, in order to achieve successful obstacle avoidance, driverless vehicles need to carry out reasonable obstacle avoidance strategy with their core functions. The core functions of driverless vehicles mainly focus on the analysis of obstacles appearing in the surrounding environment of driverless vehicles, and combine the running state of driverless vehicle fleet, choose scientific and reasonable obstacle avoidance mode, so as to complete the obstacle avoidance of driverless vehicle fleet in dynamic environment. In the actual process, there are three main ways for driverless cars to avoid obstacles: parking, following cars, changing roads. To achieve stop obstacle avoidance, mainly to make the unmanned aircraft from deceleration to static state, unmanned vehicles to achieve this function mainly rely on the braking system in the unmanned vehicle to carry out. If the driverless car meets obstacles in the course of movement, it is necessary to adjust the speed in time so that the driverless car can follow the car ahead in the course of operation, which is also one of the ways to avoid obstacles. If the driverless car meets other running conditions, it can not avoid the collision either by stopping and avoiding obstacles or by decelerating and following cars, then the driverless car can choose to change lanes to avoid obstacles, which is a relatively safe and effective obstacle avoidance measure. These three methods have different advantages and advantages, such as the way to stop and avoid obstacles, although the efficiency of obstacle avoidance is low, but such a way to avoid obstacles is safer, such as the way to avoid obstacles with cars, the speed of driverless cars has higher requirements, and the system of driverless cars has a higher standard requirements. For the third way of avoiding the obstacle of driverless car, changing the way to avoid the obstacle is the most thorough and direct way to avoid the obstacle. Therefore, the requirement of driverless car is also the highest, which requires the driverless car to have the function of strictly changing the way, and can realize the real-time route and tracking. In self-driving, driverless vehicles should be considered and analyzed strictly according to the running condition, speed and other relevant actual conditions, and judge the most suitable and safe obstacle avoidance method. In the process of choosing the obstacle avoidance method, we should not only have the requirement of high safety factor for the obstacle avoidance method, but also carry on the reasonable control grasp to the function of the driverless vehicle.

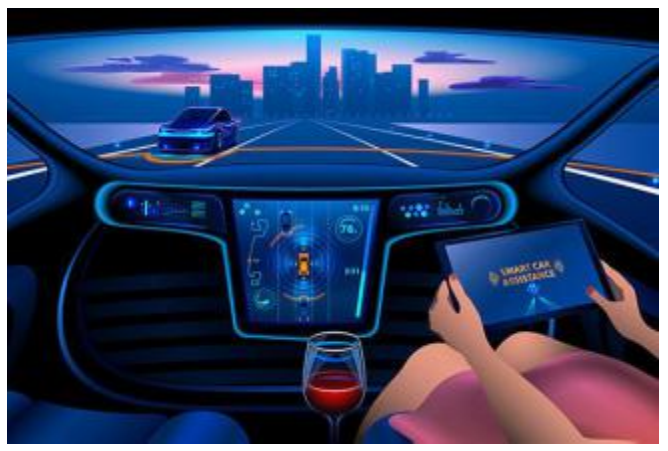

Figure 2 Driverless 


\subsection{Parking Evasion and Car-Following Evasion}

For driverless cars, adjusting the speed of driverless cars through braking function is the key to the use of parking avoidance and following car avoidance. The purpose of the adjustment of driverless cars is to keep the movement state of driverless cars consistent with the obstacles in the surrounding environment of driverless cars, and there will be no collision with obstacles in the surrounding environment of driverless cars. In the course of operation, the driverless car should always keep the proper distance from the obstacles in the surrounding environment, so that the driverless car can have sufficient time to adjust and deal with the obstacles in the process of avoiding obstacles, so as to prevent unnecessary danger. In the process of driverless vehicle braking, inertia is an inevitable influence factor. Because of the influence of inertia, it increases the instability of driverless vehicle driving. If the driverless vehicle is kept at a higher speed, too strong inertia will greatly reduce the stability of driverless vehicle and bring great discomfort to the driverless vehicle personnel. It can be seen that in order to better adjust and optimize the driverless car, to ensure that the driverless car can run smoothly at the appropriate speed on the road, can achieve a real high safety factor to avoid obstacles, The driverless car should first calculate the more accurate distance from the obstacles around the driverless car, and calculate the accurate distance according to the specific speed of the driverless car, the operating condition and the obstacles in the surrounding environment of the driverless car, and adjust the appropriate braking according to the comprehensive situation. In the process of implementing the braking function, the driverless vehicle is divided into three stages of preparation, including the braking preparation process, the unmanned vehicle starting process and the complete braking process of the driverless vehicle. The difference of the duration of these three processes has a certain influence on the braking distance of the driverless vehicle. Therefore, the system deceleration degree of driverless vehicle is divided into parking obstacle avoidance brake and following vehicle obstacle avoidance brake. For the parking armband, there is a certain limit on the obstacle avoidance braking ability of the driverless car, and the maximum deceleration of the emergency braking of the driverless car is 7 . 5 , while the deceleration of the normal braking of the driverless car is less than 4 . In the case of obstacle avoidance, the braking speed of driverless car is also within a certain range, and the minimum braking must be greater than the distance between the driverless car and the obstacle in the surrounding environment of the driverless car.

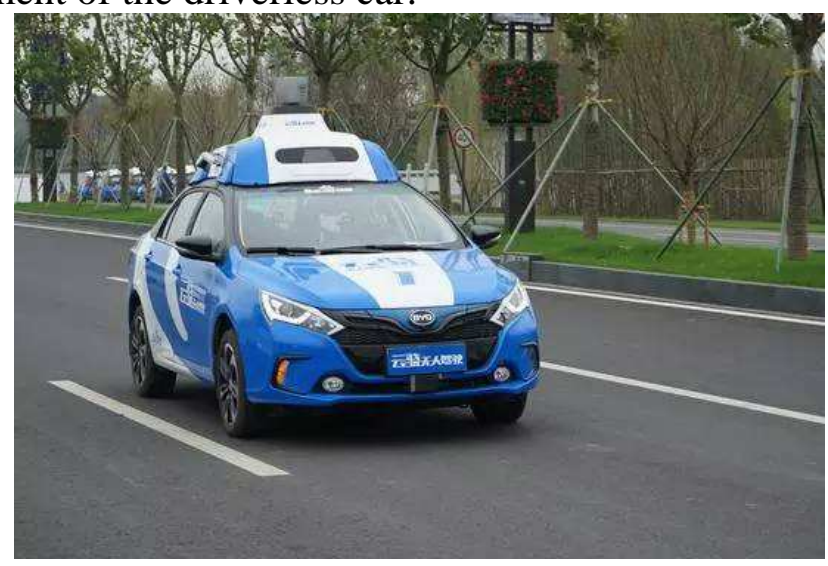

Figure 3 Driverless

\subsection{Road Access}

For driverless cars, the basic requirement of obstacle avoidance is to enable driverless cars to safely transition the adjacent lanes of the road. If the driverless car is in the form of multi-lane, it is necessary to frequently change the way to avoid obstacles. In the process of obstacle avoidance, driverless cars should choose the most suitable path, and the choice of planning path is the concept machine of obstacle avoidance. The aim of this paper is to find a way to meet the speed of the vehicle itself and to avoid obstacles well. If the relative importance of the vehicle is always unpredictable, the path selection planning should be judged according to the requirement of real 
time. In recent years, the relevant scientists in our country have strictly managed the track change, which can adapt to various situations of avoiding obstacles, but there are still some shortcomings in flexibility as a whole. The polynomial track described by the variable path obstacle can better overcome the problems of the driverless vehicle in the running process and meet the real-time requirements of the driverless vehicle in the driving process.

\section{Conclusion}

With the rapid development of our country's economic level, driverless cars are gradually accepted by people, no longer just a distant dream. In recent years, we have a deeper understanding and research on the obstacle avoidance of driverless cars. This paper analyzes the conventional obstacle avoidance methods, which may become the main obstacle avoidance mode of driverless cars. The complexity of roads and the uncertainty of obstacles have higher requirements for the obstacle avoidance of driverless cars.

\section{References}

[1] Cui, Jiachao. obstacle avoidance methods for driverless smart cars in dynamic environments .(Doctoral dissertation), 2015.

[2] Zhu, Qirong. Analysis on the Method of Avoidance of Unmanned Motor Vehicle. Technology Information, vol. 014, no. 021, pp. 53-54, 56, 2016.

[3] Huang, Xun. A Brief Introduction to the Method of Avoidance of Driverless Cars. Heilongjiang science and technology information, no. 11, 2017. 